\title{
Microwave-Triggered Surface Plasmon Coupled Chemiluminescence (MT-SPCC)
}

\author{
Michael J. R. Previte and Chris D. Geddes \\ ${ }^{1}$ Institute of Fluorescence, Laboratory for Advanced Medical Plasmonics, Medical Biotechnology \\ Center, University of Maryland Biotechnology Institute, 725 W. Lombard St., Baltimore, MD, \\ 21201. \\ RECEIVED DATE (automatically inserted by publisher); E-mail: geddes@umbi.umd.edu
}

\section{Supplementary Information}

\section{S.1 Materials}

\section{S.1.1 Materials and Reagents}

99.999\% silver evaporation slugs, and premium quality APS-coated glass slides (75x25 mm) were obtained from Sigma-Aldrich. CoverWell imaging chamber gaskets with adhesive (2.5 mm diameter, $2 \mathrm{~mm}$ deep) were obtained from Molecular Probes (Eugene, OR). Commercially available chemiluminescence materials were purchased from Unique Industries, Inc (Mississauga, Ontario).

\section{S.1.2 Chemiluminescence Reagents (Chemical reaction assays)}

The commercially available glow-sticks contain the necessary reacting chemicals, which are enclosed within a plastic tube, and yield a bright green chemiluminescent emission when they are physically altered. The plastic tube contains a phenyl oxalate ester, a fluorescent probe and a glass capsule containing the activating agent (hydrogen peroxide). Activation of the chemicals is accomplished with a bend, snap, and a vigorous shake of the plastic tube which breaks the glass capsule containing the peroxide and mixes the chemicals to begin the chemiluminescence reaction. The hydrogen peroxide oxidizes the phenyl oxalate ester to a peroxyacid ester and phenol. The unstable 
peroxyacid ester decomposes to a peroxy compound and phenol, the process chemically inducing an electronic excited state.

\section{S.2. Methods}

\section{S.2.1. Formation of Continuous Silver Films on APS-coated Glass Substrates}

Ag films were deposited on silanated glass slides by vapor deposition using an EMF Corp. (Ithaca, NY) vapor deposition instrument (Figure S1). Film thicknesses were monitored during the deposition process with an Edwards FTM6 film thickness monitor. Glass and continuous film Ag slides modified with triangle and 'bow-tie' structures were prepared by cutting equilateral $2.5 \mathrm{~mm}$ triangles in aluminum sheets with a stencil and blade (Figure S1). Disjointed 'bow-tie' geometries were constructed from two inverted $2.5 \mathrm{~mm}$ triangles, such that the distance between the apexes or gap size was approximately $1 \mathrm{~mm}$. Triangle foil masks were affixed to plain glass slides and glass slides modified with continuous Ag films $40 \mathrm{~nm}$ thick (Figure S1). Ag triangle structures were created by vapor depositing $75 \mathrm{~nm}$ of Ag films on glass and Ag slides covered with triangle masks using an EMF Corp. (Ithaca, NY) vapor deposition instrument. Film thicknesses were again monitored during the deposition process with an Edwards FTM6 film thickness monitor.

\section{S.2.2 Sample Preparation}

Glass substrates with and without continuous Ag films and substrates modified with triangle and 'bowtie' Ag structures were cut into 10 x $10 \mathrm{~mm}$ sample sizes. Image wells were placed at the tips of a single Ag triangle, between two Ag triangles, and on the unmodified Ag continuous film substrates and plain glass substrates. The samples were subsequently filled with $20 \mu \mathrm{l}$ of green chemiluminescence material. 


\section{S.2.3 Optical Configuration}

The collection optics were assembled in 1" lens tube mounts (ThorLabs, Newton, New Jersey). The lens tubes were inserted into a 1 inch diameter opening in the bottom of the microwave cavity and mounted in place using a thread adapter plate (ThorLabs, Newton, NJ), which rested inside the microwave cavity. A glass window was taped to the opening of the thread adapter plate and sat approximately $5 \mathrm{~mm}$ above the first optical element in the optical scheme. The $10 \times 10 \mathrm{~mm}$ sample geometries were affixed with index matching immersion oil to a fused silica right angle prism (Edmond Optics), whereby the prism was positioned on the glass window that covered the opening at the base of the microwave (Figure 1, left). For detection of chemiluminescence emission from the bottom of the sample, polarization measurements were performed by placing a sheet polarizer on the glass window at the base of the microwave cavity. The prism/sample geometry was placed on the sheet polarizer and data was collected with the sheet in the $p$ - or $s$ - orientation. Polarization measurements for chemiluminescence emission at the 'top' of the sample were performed by placing a sheet polarizer above the chemiluminescence solution and just below the fiber positioned above the solution.

The 'bottom' optical scheme for these experiments was configured with six BK7 AR coated plano-convex lenses (diameter $=25.4 \mathrm{~mm}$; focal length $=25.4 \mathrm{~mm}$ ) that were positioned to maximize the collection efficiency and minimize the focal spot of the surface plasmon coupled chemiluminescence. For fiber detection scheme, a collimating optic was placed in the beam path just before the fiber to maximize collection efficiency (Figure 1). For CCD camera imaging, the collimating optic was removed and the camera 
was positioned such that the emission light filled the active area of the CCD imaging sensor.

The 'top' optical scheme was configured by inserting a bare $1000 \mu \mathrm{m}$ fiber through a small hole in the top of the microwave cavity. The fiber was then positioned just above the center of the chemiluminescence solution and its output was collected using the spectrophotometer.

\section{S.2.4 Chemiluminescence from Reagents on Glass and Silver Substrates}

The chemiluminescence experiments were performed using green emission glowsticks with and without microwave heating inside the microwave cavity $(0.7 \mathrm{cu} \mathrm{ft}$, GE Compact Microwave Model: JES735BF, max power 700W). The power setting was set to 1 (10\% power). In order to obtain the same initial chemiluminescence emission for all measurements, approximately $20 \mu \mathrm{l}$ of the glow-sticks fluid was placed inside the imaging chamber. Data collection commenced 10 seconds prior to the application of the five second microwave pulse and continued until 20 seconds after microwave exposure.

Chemiluminescence spectra were collected using an Ocean Optics spectrometer, model SD 2000 (Dunedin, FL), connected to an Ocean Optics $1000 \mu$ m diameter fiber with an NA of 0.22 (Dunedin, FL). Chemiluminescent spectra and time-dependent emission intensity were collected with an integration time of 100 milliseconds. The integration time was kept constant between the control and silver island film sample measurements.

The CCD images of chemiluminescence emission on silver and glass substrates were taken with a Retiga-SRV CCD Camera (QImaging, Burnaby, B.C.) with 4x4 binning at $10 \mathrm{fps}$. 


\section{S.2.5 Data Analysis}

MT-SPCC Intensity data was collected with the spectrophotometer or CCD camera. For spectrometer measurements, peak intensity corresponds to the peak emission wavelength $(\sim 515 \mathrm{~nm})$ for the green chemiluminescence emission. Enhancement is calculated from the ratio of the maximum chemiluminescent intensity during exposure to Mw pulse to maximum emission before exposure to the Mw pulse (Figure 2, right). For polarization measurements, peak intensity for $p$ - or $s$ - polarized emission also corresponds to the peak emission wavelength ( $\sim 515 \mathrm{~nm})$ for the green chemiluminescence emission. The $p: s$ ratio of chemiluminescence emission is calculated both before exposure to Mw pulse and during exposure to the Mw pulse (Figure 2, left) 


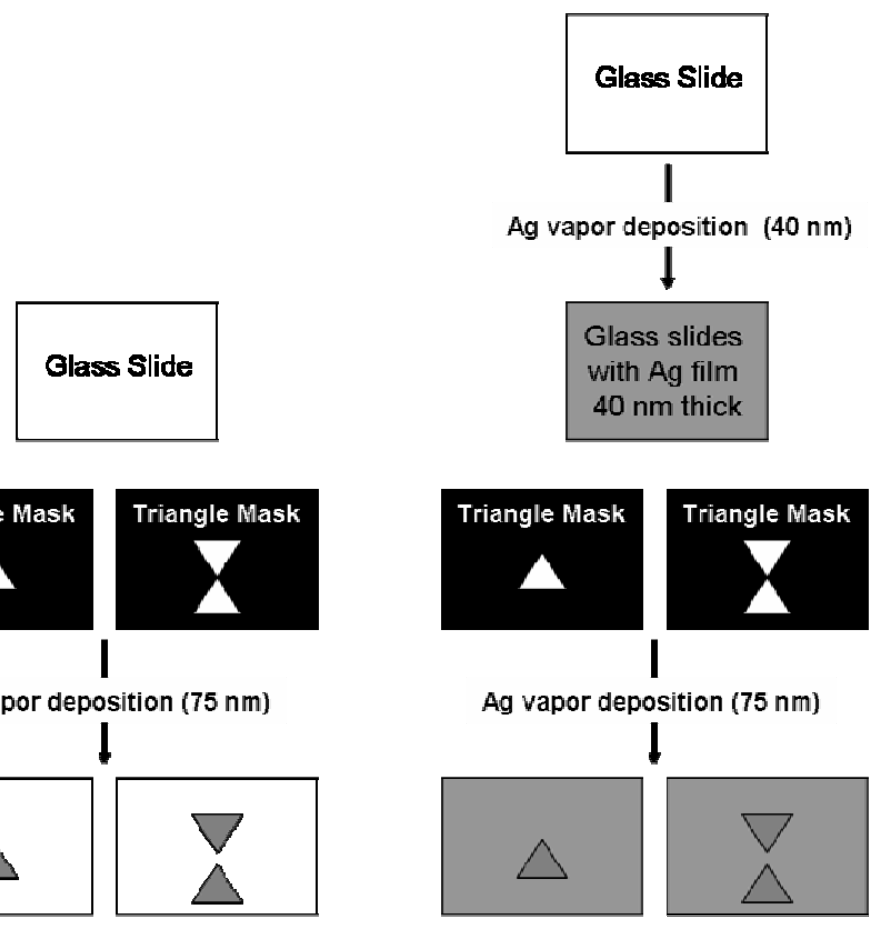

Supporting Information: Figure S1. MT-SPCC sample preparation scheme. 